\title{
Funções Neuropsicológicas Associadas a Condutas Autolesivas: Revisão Integrativa de Literatura
}

\author{
Neuropsychological Functions Associated to Self-Injurious Behavior: \\ Integrative Literature Review
}

\author{
Renata Lopes Arcoverde ${ }^{*}, a$ \& Lara Sá Leitão de Castro Soares ${ }^{b}$ \\ ${ }^{a}$ Universidade Federal de Pernambuco, Recife, Brasil \& ${ }^{b}$ Instituto de Neuropsicologia Aplicada, Recife, Brasil
}

\begin{abstract}
Resumo
Esse estudo tem como objetivo descrever fatores neuropsicológicos associados a condutas autolesivas, com base em revisão integrativa da literatura realizada através de bases de dados eletrônicas da Biblioteca Virtual em Saúde. O instrumento foi uma ficha com informações referentes a cada artigo. Foram encontrados 59 artigos e 21 (36\%) atendiam aos critérios para inclusão na amostra, que foram: artigos publicados em português ou inglês entre 2000 e 2009, disponíveis publicamente ou através do portal Periódicos Capes. Dificuldades relacionadas a resolução de problemas e tomada de decisões (38\%); impulsividade (24\%); regulação emocional $(21 \%)$ e estresse psicológico $(17 \%)$ foram fatores associados à autolesão. Os resultados sugerem correlação entre condutas autolesivas e problemas nos circuitos pré-frontais, envolvidos nos mecanismos neurais das funções citadas.

Palavras-chave: Conduta autolesiva, Neuropsicologia, córtex pré-frontal.
\end{abstract}

\begin{abstract}
This study aims to describe neuropsychological factors associated to self-injurious behavior based on an integrative literature review of papers accessed through electronic databases in the Virtual Healthcare Library. As instrument, we used information forms for each paper. A total of 59 papers were found and 21 (36\%) matched inclusion criterion: publication in Portuguese or English from 2000 to 2009 publicly available or available at CAPES Portal of Electronic Scientific Journals. Difficulties related to functions such as problem solving and decision making abilities (38\%), impulsiveness (24\%), emotional regulation $(21 \%)$ and psychological stress $(17 \%)$ were associated to self-harm. Results suggest that problems in pre-frontal circuits of the brain may be related to self-harm, since they are involved in the mechanisms of the mentioned neural functions.

Keywords: Self-injurious behavior, Neuropsychology, prefrontal cortex.
\end{abstract}

A conduta autolesiva é descrita como o ato de se machucar ou de fazer mal a si mesmo sem intenção de suicídio manifesta ou perversão sexual, através da provocação de danos físicos leves ou moderados (Kaplan, Sadock, \& Grebb, 1997). Esse comportamento é complexo e pode ter inúmeras motivações biológicas, psicológicas e/ou sociais, apresentadas com maiores detalhes na literatura. Esse estudo pretende descrever, com base em uma revisão sistemática da literatura, fatores neuropsicológicos associados a condutas autolesivas, lembrando sempre que estes são apenas mais um dos aspectos, mas não os únicos que influenciam esse tipo de comportamento (Kingdom, 2005).

As estratégias de enfrentamento do estresse, a resolução de problemas, a tomada de decisões e o controle dos

\footnotetext{
* Endereço para correspondência: Avenida Professor Moraes Rego, $1235,2^{\circ}$ andar, sala 231, Cidade Universitária, Recife, PE, Brasil 50670-901. E-mail: arcoverde.renata@gmail.com
}

impulsos são geralmente citados como importantes componentes na execução de comportamentos autodestrutivos (Bazanis, et al., 2002; Townsend et al., 2001; Withlock, Powers, \& Eckenrode, 2006). Esses componentes fazem parte das funções executivas, que são aquelas responsáveis por um conjunto de habilidades que permitem ao indivíduo planejar, direcionar seu comportamento com relação a metas determinadas, monitorar e avaliar a eficiência desses comportamentos e modificá-los caso estejam sendo ineficientes (MalloyDiniz, Sedo, Fuentes, \& Leite, 2008).

Como o fenômeno da autolesão é pouco estudado no Brasil, um obstáculo a ser superado no desenvolvimento deste estudo foi a carência de levantamento de dados brasileiros na literatura científica sobre condutas autolesivas. Assim, há a intenção de que este trabalho sirva como fonte de consulta brasileira facilmente disponível para a realização de projetos posteriores ou ampliados sobre o tema em questão. 


\section{Definições para o Comportamento Autodestrutivo}

O comportamento de provocar dano físico a si mesmo costuma causar estranheza porque contradiz o chamado instinto da busca pela sobrevivência, evitação da dor e preservação da vida. Entre estes comportamentos há aqueles socialmente aceitáveis e até mesmo legitimados, como rituais de sacrifício na passagem da adolescência à vida adulta em certas tribos ou o uso de brincos, piercings e tatuagens (Ryan, Heath, Lane, \& Young, 2008). No entanto, há situações em que essa conduta pode ser considerada como patológica, sendo objeto de estudo para pesquisadores de diversas áreas do conhecimento. Por vezes, o comportamento de provocar ferimentos em si mesmo foi chamado de para-suicídio, outras vezes de automutilação e ultimamente fala-se em autolesão deliberada ou simplesmente autolesão, porém existem algumas diferenças entre essas definições (Gratz, 2001; Hawton, Fagg, Platt, \& Hawkins, 1993; Klonsky, Oltmanns, \& Turkheimer, 2003; Runeson, Beskow, \& Waern, 1996; Yaryura-Tobias, Mancebo, \& Neziroglu, 1999). O para-suicídio é o termo mais confuso. Alguns autores dizem que esta definição supõe que há uma imitação de tentativa de suicídio, sem que haja intenção real; o indivíduo simularia essa tentativa de forma a receber atenção dos demais (Kingdom, 2005). Essa descrição está mais próxima das características da Síndrome de Münchausen, Transtorno Factício listado no DSM-IV-TR (American Psychiatric Association [APA], 2002), em que o indivíduo simula ou provoca doenças de forma a obter ganhos secundários. Outros autores usam a definição de para-suicídio como a tentativa sem êxito de suicídio, quando há a intenção do indivíduo de provocar a própria morte (Hawton et al., 1993). Esse termo não será utilizado neste projeto por provocar certa confusão, como observado acima. O simples fato de conter a palavra "suicídio" pode induzir a erros quanto a se há ou não êxito na tentativa de tirar a própria vida (Kingdom, 2005).

Já o DSM-IV TR (APA, 2002) lista a automutilação como um dos sintomas presentes para o diagnóstico do Transtorno de personalidade borderline em que o sujeito se machuca como forma de aliviar-se através da reafirmação de sua capacidade de sentir, expiar sentimentos de culpa ou evitar o abandono quando se sente ameaçado por uma separação iminente ou rejeição. Porém, mesmo em pessoas com personalidade borderline, os ferimentos podem se dar de forma superficial, suficiente apenas para provocar atitudes de cuidado de pessoas significativas (Kaplan et al., 1997). Este termo também não será utilizado aqui, pois a palavra mutilação supõe um tipo de ferimento mais grave e violento, que implicaria uma desfiguração do indivíduo. É o caso, por exemplo, do estudo feito por Nucci e Dalgalarrondo (2000), que descrevem seis casos de enucleação ocular em pacientes psicóticos. Ainda assim, há autores que utilizam o termo automutilação quando, na verdade, estão se referindo à autolesão (Richardson \& Zaleski, 1986; Yaryura-Tobias et al., 1999).
Neste estudo, adotaremos o termo autolesão, que se refere ao ato de se machucar intencionalmente de forma superficial ou moderada, com a “. . . destruição ou alteração direta do tecido corporal sem intenção suicida consciente, mas que resulte em ferimento suficientemente grave para provocar danos a esse tecido" (Gratz, 2001, p. 254), não se tratando de simulação de suicídio nem de mutilação desfigurante. Entre os atos considerados como autolesão estão: cortar-se; queimar-se; bater-se; morderse; beliscar-se; atritar objetos contra a pele; impedir ferimentos de cicatrizarem; coçar excessivamente a pele; derramar ácido, água sanitária ou outros materiais corrosivos sobre a pele; tudo isso de tal forma que ocorra o aparecimento de um ferimento (Gratz, 2001).

\section{Motivações para a Conduta Autolesiva}

De acordo com Kovács (2008), apesar de a autolesão em si não ter como motivação manifesta a intenção de suicídio, esse tipo de comportamento autodestrutivo também é uma afronta inconsciente à própria vida, assim como o abuso de drogas, acidentes freqüentes e envolvimento em outras situações arriscadas. Isso não significa que pessoas que se autolesionam tentarão algum dia o suicídio, até porque às vezes os ferimentos servem como meios de evitar a morte, através da “. . . neutralização parcial dos instintos destrutivos" (Kovács, 2008, p. 183).

Algumas motivações citadas para a autolesão são: raiva de si mesmo ou de outros, alívio de tensão, desvio do foco de atenção da dor emocional para a dor física e desejo inconsciente de morrer (Kaplan et al., 1997). Em pesquisa mais recente, realizada com mulheres jovens, sugere-se que a autolesão serve como uma âncora para a realidade, proporcionando um senso de autocontrole para pessoas com habilidades adaptativas comprometidas (Ryan et al., 2008).

Richardson e Zaleski (1986) indicam ainda que a repetição do ato de se ferir em algumas pessoas pode ser devido a uma dependência da $\beta$-endorfina, que é liberada quando há danos corporais. A liberação de $\beta$-endorfina e a sensação de dor física causada pelo ferimento funcionariam como uma forma de distração a sentimentos de angústia e frustração, causando alívio temporário. Como em qualquer relação de dependência, ocorre o fenômeno da tolerância e ferimentos cada vez mais graves são provocados para manter altos níveis de $\beta$-endorfina e evitar a sua abstinência. As explicações puramente "biologicistas" vem sendo criticadas, pois a autolesão é considerada um comportamento complexo.

Yaryura-Tobias et al. (1999) descrevem "efeitos contagiosos" em pacientes internados, indicando que o comportamento autolesivo, quando recompensado, é reforçado, e isso faz com que outros pacientes o imitem. No entanto, geralmente, a conduta autolesiva é motivo de vergonha para os indivíduos, que chegam a esconder os ferimentos de amigos e familiares. Dessa forma, é possível que as taxas de prevalência de autolesão sejam subes- 
timadas (Puskar et al., 2006). A prevalência de autolesão é maior entre mulheres (Kingdom, 2005); adolescentes e jovens adultos (Rodham, Hawton, \& E. Evans, 2004), prisioneiros (Caldas et al., 2010) e pacientes psiquiátricos (Gratz, 2001), notadamente naqueles com personalidade borderline, transtornos alimentares, transtorno de estresse pós-traumático, depressão, transtornos de ansiedade e aqueles com um histórico de abuso ou trauma (Withlock et al., 2006). Com relação à ocorrência da autolesão, o ato de se machucar propositalmente tem sido descrito como uma manifestação independente, e não apenas como um sintoma de transtornos psiquiátricos, visto que este tipo de conduta é encontrado em uma variedade diversa de diagnósticos e mesmo em populações não-clínicas (Klonsky et al., 2003).

\section{Correlação com Funções Neuropsicológicas}

Vários autores (Bazanis et al., 2002; Townsend et al., 2001; Withlock et al., 2006) indicam que condutas autolesivas sugerem certo comprometimento no controle dos impulsos e na tomada de decisões, assim como na elaboração de estratégias para lidar com situações de estresse e resolver problemas. Essas funções estão relacionadas ao córtex pré-frontal, especificamente o córtex órbitofrontal, envolvido no planejamento de ações, raciocínio, tomada de decisões, resolução de problemas e ajuste social do comportamento (Lent, 2005).

Malloy-Diniz et al. (2008) comentam que o controle inibitório é a função neuropsicológica que confere a habilidade para interromper o impulso de dar uma resposta ou efetuar uma ação específica diante de determinado estímulo. Essa resposta geralmente é de caráter prepotente, ou seja, se apresenta como de forte tendência para o indivíduo. São os problemas nos circuitos órbito-frontais que implicam em dificuldades para superar tendências imediatistas e considerar conseqüências em longo prazo (Malloy-Diniz, Jardim, LoschiavoAlvares, Fuentes, \& Leite, 2010). Esse pode ser o caso das condutas autolesivas impulsivas.

Por sua vez, as funções de tomada de decisões e resolução de problemas envolvem o processo de escolher uma dentre várias alternativas de ação, considerando suas vantagens e desvantagens em termos de resultados (se a alternativa será eficiente para alcançar a meta pretendida), aspectos sociais ou morais (se a alternativa é vista como socialmente ou moralmente inaceitável para o sujeito ou para a sociedade) e autoconsciência - viabilidade e possibilidades pessoais de arcar com a escolha (Malloy-Diniz et al., 2008).

Neste caso, pode-se considerar a conduta autolesiva como uma alternativa de ação utilizada como estratégia de escapismo ou de enfrentamento do estresse: ao se encontrar numa situação de sofrimento psíquico, o indivíduo se fere propositalmente, pois a dor física desvia o foco desse sofrimento, resolvendo o problema, ainda que temporariamente (Kaplan et al., 1997). Tal ligação entre o emocional e o físico pode ser explicada, pois o córtex pré-frontal mantém contato direto com algumas estruturas do sistema límbico envolvidas no processamento emocional, como o hipotálamo, o hipocampo e a amígdala. Assim, disfunções nos mecanismos de regulação emocional também podem ser fatores correlacionados a comportamentos autolesivos. O hipotálamo é tido como um centro de controle das manifestações fisiológicas que acompanham as emoções e o hipocampo não participa diretamente dos mecanismos neurais da emoção, mas age na consolidação das memórias, inclusive daquelas com conteúdo emocional (Lent, 2005).

\section{Método}

\section{Tipo de Estudo}

O presente estudo é uma revisão integrativa da literatura, que consiste em reunir pesquisas já realizadas sobre determinada questão, de forma a alcançar uma maior compreensão sobre o tema estudado (Whittemore \& Knafl, 2005). Castro (2002) informa que as revisões de literatura, de forma geral, podem oferecer diretrizes clínicas úteis para os tomadores de decisão na área da saúde, sendo importantes para profissionais e gestores, além de poder contribuir para o planejamento de futuras pesquisas clínicas.

\section{Locais de Busca}

Os artigos foram acessados através da Biblioteca Virtual em Saúde (BVS) e estavam publicamente disponíveis ou disponíveis através de acesso ao portal de periódicos oferecido pela Coordenação de Aperfeiçoamento do Pessoal de Nível Superior (CAPES), que proporciona acesso a textos completos de artigos em revistas nacionais e internacionais.

\section{População e Amostra}

Os critérios para inclusão na amostra foram: abordagem das temáticas: condutas autolesivas e funções neuropsicológicas; trabalhos publicados entre os anos de 2000 e 2009 e publicação em língua portuguesa ou inglesa. E os critérios de exclusão foram: artigos de acesso pago, não disponíveis nos Periódicos CAPES ou outro site de acesso público e gratuito; artigos repetidos na busca com diferentes descritores e temas incompatíveis com o objeto de estudo. Considerou-se tanto estudos primários como de revisão de literatura.

Os temas incompatíveis estavam relacionados com os descritores utilizados para a busca, porém não diziam respeito ao objeto de estudo especificado para a revisão, que é a correlação entre autolesão e neuropsicologia. Entre os temas não inclusos estão: suicídio sem correlação com autolesão; automutilação grave/amputação; atitudes da equipe de saúde frente ao paciente que se autolesiona; questões jurídicas sobre o comportamento autodestrutivo. 


\section{Instrumento para Coleta de Dados}

$\mathrm{O}$ instrumento utilizado foi uma ficha para anotação das informações referentes a cada trabalho selecionado para composição da amostra. A ficha contém os seguintes itens a serem preenchidos: título do artigo; ano da publicação; título do periódico; descritores; resumo do artigo.

\section{Operacionalização da Coleta de Dados}

Entre setembro e outubro de 2009, artigos sobre condutas autolesivas associadas a funções neuropsicológicas foram coletados nas bases de dados eletrônicas. Todos eles foram avaliados, de forma a selecionar aqueles que obedeciam aos critérios para inclusão no estudo. Os descritores foram selecionados a partir do vocabulário estruturado Descritores em Ciências da Saúde ([DeCS], BVS, 2008).

Assim, foram utilizados os seguintes descritores, nos idiomas português e inglês: Comportamento Autodestrutivo/Self-Injurious Behavior; Automutilação/Self Mutilation; Tomada de Decisões/Decision Making; Resolução de Problemas/Problem Solving; Comportamento Impulsivo/Impulsive Behavior; Estresse Psicológico/ Stress, Psychological; Neuropsicologia/Neuropsychology; Testes Neuropsicológicos/Neuropsychological Tests.

Ainda que as autoras deste estudo compreendam que o termo mais adequado para o tipo de comportamento em questão seria autolesão, o termo automutilação é listado como descritor. Essa inclusão se justifica pois, em revisões, é necessário considerar a variação de definições entre os autores quando estes se referem ao mesmo objeto (Castro, 2002). Assim, quando se encontrava um artigo com o descritor automutilação, avaliava-se se o mesmo realmente versava sobre ferimentos mais graves ou se, na realidade, os autores se referiam à autolesão.

\section{Apresentação e Análise dos Dados}

Os dados coletados estão organizados em uma tabela e analisados quantitativamente, através de estatística descritiva. Os resultados foram categorizados por tema (resolução de problemas e tomada de decisões; impulsividade; regulação emocional e estresse psicológico), após a leitura dos títulos, resumos e textos dos artigos selecionados, de forma a permitir a análise à luz da literatura especializada sobre a questão.

\section{Resultados e Discussão}

Com a utilização dos descritores e a verificação de acessibilidade pública ou através dos Periódicos CAPES, foram encontrados 59 artigos. Desses, apenas 21 (36\%) foram selecionados para a amostra após a avaliação dos critérios de inclusão. Os restantes foram excluídos, pois 15 apresentavam publicação anterior ao ano 2000 (25\%); 20 abordavam temas incompatíveis com a proposta do estudo $(34 \%)$ e três $(5 \%)$ foram publicados em idioma diferente do português ou inglês.
Consideramos que o total de artigos que relacionam a conduta autolesiva a fatores neuropsicológicos é baixo, talvez porque a autolesão é um tema de difícil estudo, visto que os indivíduos que apresentam esse tipo de conduta não são facilmente identificados. Muitas vezes, eles têm vergonha e escondem seus ferimentos ou mentem sobre eles. Assim, há uma dificuldade de recrutar essas pessoas para participar de pesquisas (Puskar et al., 2006).

Todos os artigos encontrados para a amostra foram escritos no idioma inglês. Isso reforça o que já havia sido dito sobre a falta de dados brasileiros sobre a autolesão (Caldas et al., 2010). Dessa forma, acreditamos que o presente trabalho poderá ser uma fonte útil para aqueles que se interessarem pelo tema e desejarem consultar um estudo em português.

A Tabela 1 apresenta a categorização por tema dos artigos selecionados. O total da freqüência é maior do que a quantidade de artigos selecionados, pois, às vezes, o mesmo artigo abordava mais de um tema diferente. Além disso, a divisão por categorias é apenas didática, visto que elas estão em constante inter-relação e não se pode considerá-las isoladamente.

Tabela 1

Categorização por Tema dos Artigos Selecionados

\begin{tabular}{lcc}
\hline Tema & Freqüência & $\%$ \\
\hline Resolução de problemas & & \\
e tomada de decisões & 11 & 38 \\
Impulsividade & 7 & 24 \\
Regulação emocional & 6 & 21 \\
Estresse psicológico & 5 & 17 \\
Total & 29 & 100 \\
\hline
\end{tabular}

Ao total, os estudos envolveram 4605 participantes. Entre aqueles com condutas autolesivas foram encontrados mulheres, adolescentes e jovens adultos, com transtornos diversos, tais como: personalidade borderline, depressão, transtornos alimentares e pessoas da população geral, sem diagnóstico psiquiátrico. Essa prevalência está de acordo com os dados indicados na literatura especializada sobre quem pratica autolesão (Gratz, 2001; Kingdom, 2005; Klonsky et al., 2003; Rodham et al., 2004; Withlock et al., 2006).

\section{Resolução de Problemas e Tomada de Decisões}

Foram encontradas onze referências (38\%) que associavam autolesão a resolução de problemas e tomada de decisões. No total, esses estudos contaram com uma amostra de 1584 sujeitos. Constatou-se que pessoas que apresentavam condutas autolesivas tinham sua capacidade para a resolução de problemas prejudicada, em comparação com sujeitos que não apresentavam esse comportamento, e supõe-se que esse prejuízo interfere na escolha de respostas sociais mais adequadas (Mcauliffe, 
Corcoran, Hickey, \& Mcleavey, 2008; Milnes, Owens, \& Blenkiron, 2002; Nock \& Mendes, 2008; Oldershaw et al., 2009). Um estudo brasileiro observou que, em relação à flexibilidade na resolução de problemas, pessoas que tentaram o suicídio também apresentavam pior desempenho do que os sujeitos controle nos testes de Wisconsin e Stroop. As autoras acreditam que isso evidencia uma deficiência nessa habilidade e sugerem uma melhor investigação quanto a um possível comprometimento pré-frontal (Keller \& Werlang, 2005).

Nock e Mendes (2008) indicam ainda que podem ocorrer várias disfunções na seqüência de processamento de informações que influenciam comportamentos considerados como não adequados socialmente, tais como a autolesão e a tentativa de suicídio. Por exemplo, pode haver problemas na interpretação das informações vindas do ambiente. Nesse caso, os indivíduos veriam no comportamento dos outros uma crítica a eles mesmos e utilizariam a autolesão como forma de punição. Por outro lado, segundo os mesmos autores, é possível que essas pessoas sejam até capazes de gerar soluções eficazes para a resolução de problemas, mas acabem por selecionar aquelas consideradas como não adaptativas (nesse caso, podemos incluir tanto a tentativa de suicídio como a autolesão).

Essa decisão pode ser influenciada pelo fato de que, em geral, os indivíduos que se engajam em tais condutas apresentam baixa auto-estima e desesperança, não acreditando que existam alternativas mais eficazes para lidar com seus problemas (Keller \& Werlang, 2005). Podemos supor que o mesmo mecanismo utilizado para descrever o que ocorre com tentadores de suicídio pode ser citado com relação à conduta autolesiva, pois tratamentos baseados na melhoria de habilidades para a resolução de problemas são apontados como benéficos na redução desse comportamento (Klonsky \& Muehlenkamp, 2007; Mcauliffe et al., 2006; Mcauliffe et al., 2008; Milnes et al., 2002; Nock \& Mendes, 2008; Oldershaw et al., 2009; Slee, Garnefski, Van Der Leeden, Arensman, \& Spinhoeven, 2008).

\section{Comportamento Impulsivo}

Sete artigos (24\%) associavam autolesão a impulsividade. $\mathrm{O}$ total de sujeitos na amostra desses artigos foi de 1506 pessoas. J. Evans et al. (2000) encontraram altos índices de impulsividade em pacientes com condutas autolesivas, em comparação com indivíduos da população em geral. Da mesma forma, Berlin e Rolls (2004) apontam para uma correlação entre impulsividade e problemas associados ao córtex órbito-frontal em pacientes com transtorno de personalidade borderline e comportamento autolesivo. Esse dado está de acordo com outra pesquisa, que verificou o desempenho de pacientes com as mesmas características acima em testes computadorizados de tomada de decisões e planejamento. Em geral, os participantes apresentaram um padrão de escolhas não adaptativas e impulsivas (Bazanis et al., 2002). A impulsividade, neste caso, está ligada à tomada de decisões e acontece por falta de planejamento, pois o indivíduo não utiliza um tempo para analisar possíveis alternativas de resposta e as conseqüências de seu ato no futuro, agindo por impulso.

Já Rodham et al. (2004) analisaram adolescentes da comunidade e os separaram em dois grupos: aqueles que se feriam através de cortes na pele e aqueles que tomaram overdoses de substâncias intencionalmente. Aqueles que se cortavam afirmaram com maior freqüência que passaram menos de uma hora planejando o ato. Segundo os autores, nesses casos é possível haver maior influência da impulsividade do que no caso dos que tomaram overdoses, pois é mais fácil ferir-se através de cortes com o que estiver à mão no momento, enquanto que para ferir-se através de um envenenamento seria preciso um maior planejamento.

Um tipo diferente de autolesão, não citado até o momento, foi mencionado por Favaro e Santonastaso (2000), que consideram que o comportamento de purgação encontrado em pacientes com bulimia nervosa pode ser visto como uma forma impulsiva de autopunição.

Por fim, há uma referência ao comprometimento da microestrutura de matéria branca em regiões frontais inferiores do cérebro de mulheres com transtorno de personalidade borderline e conduta autolesiva. Esse comprometimento pode estar relacionado à incapacidade de equilibrar o alívio imediato de tensão após o ato de se ferir e o reconhecimento das conseqüências desse ato em longo prazo (Grant et al., 2007).

\section{Regulação Emocional}

Seis artigos (21\%), com um número total de 364 participantes, citavam a regulação emocional como um importante aspecto a ser analisado na conduta autolesiva. Jallade, Sarfati e Hardy-Baylé (2005) descreveram o que parece ser um efeito catártico em indivíduos que se autoagrediram, logo após o ato. Esse efeito funcionaria como um meio de aliviar a tensão psicológica e poderia ser uma conseqüência do apoio recebido da equipe médica e da família ou amigos. Já Kamphuhis, Ruyling e Reijtjes (2007) examinaram o estado emocional de mulheres antes e depois de terem cometido um ato autolesivo. Emoções consideradas como negativas apresentavam-se com maior intensidade imediatamente antes da autolesão, diminuindo significativamente logo após o ato e aumentando mais uma vez um dia depois. O inverso acontecia com emoções consideradas como positivas pelas participantes. Da mesma forma, Paul, Schroeter, Dahme e Nutzinger (2002) encontraram 69,2\% de mulheres com transtornos alimentares que afirmaram sentir-se melhor logo após se ferirem. No entanto, apenas $33 \%$ continuava a sentir-se bem após um maior intervalo de tempo.

Esses resultados corroboram o que dizem vários autores quando afirmam que o comportamento autolesivo pode ser praticado como forma de aliviar emoções negativas, ainda que temporariamente (Kamphuhis et al., 2007; 
Kaplan et al., 1997; Kovács, 2008; Ryan et al., 2008). Quanto a essa sensação apenas temporária de bem-estar, podemos lembrar o mecanismo citado por Richardson e Zaleski (1986), que apontam a repetição da autolesão como um sistema que envolve um círculo vicioso: o dano corporal causa a liberação de $\beta$-endorfina, que provoca a sensação de bem-estar. Porém, a repetição do dano causa o fenômeno da tolerância e é necessária uma quantidade cada vez maior de $\beta$-endorfina para que o mesmo efeito ocorra, o que caracterizaria uma dependência dessa substância, com repetição da conduta autolesiva.

Outro artigo citou a dor como fator associado ao comportamento autolesivo em mulheres com transtorno de personalidade borderline que se machucavam intencionalmente. Constatou-se que $67 \%$ delas apresentavam uma percepção da dor mais atenuada em relação a sujeitos controle (Schmahl et al., 2004). Esse dado corrobora outros estudos que mostram que o alívio da dor emocional parece se sobrepor à dor física. Em uma população de detentas da Colônia Penal Feminina do Recife, 68\% das mulheres que apresentavam condutas autolesivas relataram não sentir dor, não se tratando, portanto, de um comportamento masoquista (Caldas et al., 2010).

Por fim, Slee, Spinhoven, Garnefski e Arensman (2008) sugerem que as intervenções terapêuticas a serem realizadas com indivíduos que se autolesionam não devem ser focadas primariamente nos transtornos associados a esse comportamento, mas devem ser específicas e enfatizar as dificuldades de regulação emocional. Assim, podese também pensar na possibilidade de investigação da atuação do sistema límbico e dos circuitos que o conectam com o córtex pré-frontal nesses indivíduos, visto que essas regiões englobam os mecanismos de regulação das emoções. A sugestão está coerente com as taxas de prevalência de autolesão encontradas na literatura especializada, já que esse comportamento pode ser visto como um fenômeno por si só, sendo encontrado em associação com diversos transtornos e até em populações não-clínicas, segundo apontado por Klonsky et al. (2003).

\section{Estresse Psicológico}

Cinco artigos (17\%), que reuniram 1151 sujeitos, citaram o estresse psicológico como fator associado à autolesão. Indivíduos com esse comportamento experimentariam uma maior excitação fisiológica após eventos estressantes e utilizariam a autolesão como forma de lidar com o estresse, um mecanismo de enfrentamento semelhante ao exposto no tópico sobre regulação emocional (Nock \& Mendes, 2008). Além disso, a memória autobiográfica também foi citada como aspecto a ser observado, pois memórias estressantes podem atuar como gatilho para a prática da autolesão (Startup et al., 2001). A esse respeito, podemos pensar na atuação conjunta do lobo pré-frontal (envolvido na tomada de decisões e resolução de problemas), do sistema límbico (responsável pelas emoções) e das memórias. É possível que a recordação de eventos carregados de emoções negativas de- sencadeie a resposta de autolesão para lidar com o estresse. Uma vez que esse circuito é consolidado pelo sujeito, ele é aprendido e torna-se o padrão de resposta comportamental. Assim, podemos pensar também na atuação da amígdala, que parece padronizar as respostas comportamentais apropriadas para cada ocasião e faz esse papel de gatilho emocional (Lent, 2005).

O estresse também pode ser percebido sem que exista uma situação externa. Desesperança, depressão, ansiedade e angústia são manifestações de estresse da mesma forma, ainda que percebidos como internos ao indivíduo (Hilt, Cha, \& Nolen-Hoeksema, 2008). Keller e Werlang (2005, p. 130) dizem o seguinte:

Sujeitos com história de tentativa de suicídio apresentavam rigidez cognitiva, pois não possuiriam habilidades diversas para o enfrentamento de situações estressantes, não conseguindo identificar soluções alternativas para resolver o problema, situação descrita anteriormente como característica de adolescentes com comportamento autodestrutivo.

Assim, a ansiedade e a depressão podem estar correlacionadas à autolesão, pois além de eliciar emoções e sentimentos negativos, também afetam o desempenho do indivíduo em tarefas que envolvem a participação das funções executivas. A depressão está associada a alterações de desatenção e pouca flexibilidade cognitiva, enquanto que a ansiedade implica menores níveis de tolerância à frustração e maior impulsividade (Fuentes, Malloy-Diniz, Camargo, \& Cosenza, 2008; Lent, 2005).

\section{Conclusões}

Através de uma revisão da literatura, foi possível descrever alguns dos fatores neuropsicológicos associados a condutas autolesivas, no entanto, o total de estudos encontrados é considerado baixo. Um dos motivos para isso pode ser a dificuldade de identificar pessoas dispostas a participar de pesquisas, pois muitos escondem o comportamento autolesivo, por vergonha ou medo de represálias (Puskar et al., 2006). Assim, os resultados apresentados não devem ser generalizados e se faz necessário avaliar a forma de estudar o fenômeno para se chegar a conclusões mais ampliadas e talvez sugerir intervenções.

Primeiramente, verificou-se dificuldades na resolução de problemas e tomada de decisões das pessoas que se autolesionam. Supõe-se que esse prejuízo interfere na escolha de respostas sociais mais adequadas porque os indivíduos interpretam erroneamente informações vindas do ambiente, vendo no comportamento dos outros uma crítica a eles mesmos e utilizando práticas autolesivas como forma de autopunição (Nock \& Mendes, 2008). Outra suposição é que mesmo que essas pessoas consigam gerar soluções eficazes para a resolução de problemas, elas tomam a decisão de selecionar com maior freqüência respostas consideradas como não adaptativas, pois, em geral, os indivíduos que se engajam em tais condutas apresentam sentimentos de desesperança e baixa 
auto-estima, não acreditando que existam alternativas mais eficazes para lidar com seus problemas (Keller \& Werlang, 2005). Dessa forma, sugere-se a realização de investigações mais aprofundadas para verificar possíveis disfunções em regiões pré-frontais do cérebro.

Além disso, estudos mostram prejuízos na tarefa de planejamento, pois o indivíduo não utiliza um tempo para analisar as possíveis alternativas de resposta e as conseqüências de seu ato no futuro, agindo por impulso. A esse respeito, comenta-se que um comprometimento da microestrutura de matéria branca em regiões frontais inferiores do cérebro pode estar relacionado à incapacidade de equilibrar o alívio imediato de tensão após o ato de se ferir e o reconhecimento das conseqüências desse ato em longo prazo (Gratz, 2001).

Problemas no mecanismo de regulação emocional, envolvendo as conexões entre o sistema límbico e o córtex pré-frontal, podem estar associados à autolesão. Apesar de se sentirem temporariamente mais aliviados logo após o ato, os sentimentos negativos voltam a aparecer e o ciclo se repete (Kamphuhis et al., 2007; Kaplan et al., 1997; Kovács, 2008; Ryan et al., 2008).

Lembranças estressantes, ansiedade ou depressão poderiam atuar como gatilho para ativar esse mecanismo disfuncional de regulação das emoções e ajudar a consolidar a autolesão como um padrão de resposta. Assim, além de envolver a memória autobiográfica e o hipotálamo, esse mecanismo pode também envolver a atuação da amígdala, que regula essa função de gatilho emocional e padronização de respostas comportamentais (Lent, 2005; Startup et al., 2001).

Pode-se notar que essas considerações, para além do enfrentamento do estresse, também envolvem o papel da capacidade de resolução de problemas e da regulação emocional, o que reforça a inter-relação entre as diversas funções neuropsicológicas, aqui apresentadas separadamente apenas para fins didáticos e de classificação dos artigos encontrados a partir dos descritores propostos. Estudos futuros podem esclarecer melhor o papel específico das estruturas pré-frontais e límbicas no ativamento do gatilho para a conduta autolesiva, além de considerar os aspectos psicossociais presentes na vida dos indivíduos que cometem autolesão.

\section{Referências}

American Psychiatric Association. (2002). DSM-IV-TR: Manual diagnóstico e estatístico de transtornos mentais (4. ed. rev.). Porto Alegre, RS: Artmed.

Bazanis, E., Rogers, R. D., Dowson, J. H., Taylor, P., Meux, C., Stanley, C., et al. (2002). Neurocognitive deficits in decision-making and planning of patients with DSM-III-R borderline personality disorder. Psychological Medicine, 32(8), 1395-1405.

Berlin, H. A., \& Rolls, E. T. (2004). Time perception, impulsivity, emotionality, and personality in self-harming borderline personality disorder patients. Journal of Personality Disorders, 18(4), 358-378.
Biblioteca Virtual em Saúde. (2008). DeCS - Descritores em Ciências da Saúde. São Paulo, SP: Autor. Retrieved August 4, 2009, from http://decs.bvs.br/

Caldas, M. T., Arcoverde, R. L., Santos, T. F., Lima, M. S., Macedo, L. E. M. L., \& Lima, M. C. (2010). Condutas autolesivas entre detentas da Colônia Penal Feminina do Recife. Psicologia em Estudo, 14(3), 575-582.

Castro, A. A. (Ed.). (2002). Curso de revisão sistemática e metanálise. São Paulo, SP: Universidade Federal de São Paulo. Retrieved July 31, 2009, from http://www.virtual.epm.br/ cursos/metanalise

Evans, J., Reeves, B., Platt, H., Leibenau, A., Goldman, D., Jefferson, K., et al. (2000). Impulsiveness, serotonin genes and repetition of deliberate self-harm. Psychological Medicine, 30(6), 1327-1334.

Favaro, A., \& Santonastaso, P. (2000). Self-Injurious behavior in anorexia nervosa. Journal of Nervous and Mental Disease, 188(8), 537-542.

Fuentes, D., Malloy-Diniz, L. F., Camargo, C. H. P., \& Cosenza, R. M. (Eds). (2008). Neuropsicologia: Teoria e prática. Porto Alegre, RS: Artmed.

Grant, J. E., Correia, S., Brennan-Krohn, T., Malloy, P. F., Laidlaw, D. H., \& Schulz, S. C. (2007). Frontal white matter integrity in borderline personality disorder with self-injurious behavior. Journal of Neuropsychiatry and Clinical Neurosciences, 19(4), 383-390.

Gratz, K. L. (2001). Measurement of deliberate self-harm: Preliminary data on the deliberate self-harm inventory. Journal of Psychopathology and Behavioral Assessment, 23(4), 253-263.

Hawton, K., Fagg, J., Platt, S., \& Hawkins, M. (1993). Factors associated with suicide after parasuicide in young people. British Medical Journal, 306, 1641-1644.

Hilt, L. M., Cha, C. B., \& Nolen-Hoeksema, S. (2008). Nonsuicidal self-injury in young adolescent girls: Moderators of the distress-function relationship. Journal of Consulting and Clinical Psychology, 76(1), 63-71.

Jallade, C., Sarfati, Y., \& Hardy-Baylé, M.-C. (2005). Clinical evolution after self-induced or accidental traumatism: A controlled study of the extent and the specificity of suicidal catharsis. Journal of Affective Disorders, 85(3), 283-292.

Kamphuhis, J. H., Ruyling, S. B., \& Reijntjes, A. H. (2007). Testing the emotion regulation hypothesis among self-injuring females: Evidence for differences across mood states. Journal of Nervous and Mental Disease, 195(11), 912-918.

Kaplan, H. I., Sadock, B. J., \& Grebb, J. A. (1997). Compêndio de Psiquiatria: Ciencias do comportamento e Psiquiatria Clínica. Porto Alegre, RS: Artmed.

Keller, M., \& Werlang, B. S. G. (2005). Flexibilidade na resolução de problemas em tentadores de suicídio. Jornal Brasileiro de Psiquiatria, 54(2), 128-136.

Kingdom, A. (2005). Self-injurious behaviour and deliberate self-harm. In T. Riding, C. Swann, \& B. Swann (Eds.), The handbook of forensic learning disabilities (pp. 97-120). London: Radcliff Pub. Retrieved August 5, 2009, from http:/ /books.google.com/books?hl=pt-BR\&lr=\&id=ziX 6UAkt MMC\&oi $=$ fnd \&pg $=$ PA $97 \& d q=$ parasuicide + munchausen\&ots $=\mathrm{eZnZFH} 2 \mathrm{CkS} 8 \& \mathrm{sig}=\mathrm{tsF}$ oqHqJnr GqmdHs2AM4Up8zWVk\#PPA97,M1

Klonsky, D. E., \& Muehlenkamp, J. J. (2007). Self-injury: A research review for the practitioner. Journal of Clinical Psychology, 63(11), 1045-1056. 
Klonsky, D. E., Oltmanns, T. F., \& Turkheimer, E. (2003) Deliberate self-harm in a nonclinical population: Prevalence and psychological correlates. American Journal of Psychiatry, 160(8), 1501-1508.

Kovács, M. J. (2008). Comportamentos autodestrutivos e o suicídio. In M. J. Kovács (Ed.), Morte e desenvolvimento humano (pp. 171-194). São Paulo, SP: Casa do psicólogo.

Lent, R. (2005). Cem bilhões de neurônios: Conceitos fundamentais de Neurociência. São Paulo, SP: Atheneu.

Malloy-Diniz, L. F., Jardim, J. P., Loschiavo-Alvares, F. Q., Fuentes, D., \& Leite, W. B. (2010). Exame das funções executivas. In L. F. Malloy-Diniz, D. Fuentes, P. Mattos, \& N. Abreu (Eds.), Avaliação neuropsicológica (pp. 94-113), São Paulo, SP: Artmed.

Malloy-Diniz, L. F., Sedo, M., Fuentes, D., \& Leite, W. B. (2008). Neuropsicologia das funções executivas. In D. Fuentes, L. F. Malloy-Diniz, C. H. P. Camargo, \& R. M. Cosenza (Eds.), Neuropsicologia: Teoria e prática (pp. 187206). Porto Alegre, RS: Artmed.

Mcauliffe, C., Corcoran, P., Hickey, P., \& Mcleavey, B. C. (2008). Optional thinking ability among hospital-treated deliberate self-harm patients: A I -year follow-up study. British Journal of Clinical Psychology, 47(1), 43-58.

Mcauliffe, C., Corcoran, P., Keeley, H. S., Arensman, E., BilleBrahe, U., De Leo, D., et al. (2006). Problem-solving ability and repetition of deliberate self-harm: A multicentre study. Psychological Medicine, 36(1), 45-55.

Milnes, D., Owens, D., \& Blenkiron, P. (2002). Problems reported by self-harm patients: Perception, hopelessness and suicidal intent. Journal of Psychosomatic Research, 53(3), 819-822.

Nock, M. K., \& Mendes, W. B. (2008). Physiological arousal, distress tolerance, and social problem-solving deficits among adolescent self-injurers. Journal of Consulting and Clinical Psychology, 76(1), 23-38.

Nucci, M. G., \& Dalgalarrondo, P. (2000). Automutilação ocular: Relato de seis casos de enucleação ocular. Revista Brasileira de Psiquiatria, 22(2), 80-86.

Oldershaw, A., Grima, E., Jollant, F., Richards, C., Simic, M., Taylor, L., et. al. (2009). Decision making and problem solving in adolescents who deliberately self-harm. Psychological Medicine, 39(1), 95-104.

Paul, T., Schroeter, K., Dahme, B., \& Nutzinger, D. O. (2002). Self-injurious behavior in women with eating disorders. American Journal of Psychiatry, 159(3), 408-411.

Puskar, R. K., Bernardo, L., Hatam, M., Geise, S., Bendik, J., \& Grabiak, B. R. (2006). Self-cutting behaviors in Adolescents. Journal of Emergency Nursing, 32(5), 444-446.

Richardson, J. S., \& Zaleski, W. A. (1986). Endogenous opiates and self-mutilation. American Journal of Psychiatry, 143(7), 938-939.

Rodham, K., Hawton, K., \& Evans, E. (2004). Reasons for deliberate self-harm: Comparison of self-poisoners and selfcutters in a community sample of adolescents. Journal of the American Academy of Child \& Adolescent Psychiatry, 43(1), 80-87.

Runeson, B. S., Beskow, J., \& Waern, M. (1996). The suicidal process in suicides among young people. Acta Psychiatrica Scandinavica, 93(1), 35-42.
Ryan, K., Heath, M. A., Lane, F., \& Young, E. L. (2008). Superficial self-harm: Perceptions of young women who hurt themselves. Journal of Mental Health Counseling, 30(3), 237-254.

Schmahl, C., Greffrath, W., Baumgärtner, U., Schlereth, T., Magerl, W., Philipsen, A., et al. (2004). Differential nociceptive deficits in patients with borderline personality disorder and self-injurious behavior: Laser-evoked potentials, spatial discrimination of noxious stimuli, and pain ratings. Pain, 110(1/2), 470-479.

Slee, N., Garnefski, N., Van Der Leeden, R., Arensman, E., \& Spinhoven, P. (2008) Cognitive-behavioural intervention for self-harm: Randomised controlled trial. British Journal of Psychiatry, 192(3), 202-211.

Slee, N., Spinhoven, P., Garnefski, N., \& Arensman, E. (2008). Emotion regulation as mediator of treatment outcome in therapy for deliberate self-harm. Clinical Psychology and Psychotherapy, 15(4), 205-216.

Startup, M., Heard, H., Swales, M., Jones, B., Williams, J. M. G., \& Jones, R. S. P. (2001). Autobiographical memory and parasuicide in borderline personality disorder. British Journal of Clinical Psychology, 40(2), 113-120.

Townsend, E., Hawton, K., Altman, D. G., Arensman, E., Gunnell, D., Hazell, P., et al. (2001). The efficacy of problemsolving treatments after deliberate self-harm: Meta-analysis of randomized controlled trials with respect to depression, hopelessness and improvement in problems. Psychological Medicine, 31(6), 979-988.

Whittemore, R., \& Knafl, K. (2005). The integrative review: updated methodology. Journal of Advanced Nursing, 52(5), 546-553.

Withlock, J. L., Powers, J. L, \& Eckenrode, J. (2006). The virtual cutting edge: The internet and Adolescent self-injury. Developmental Psychology, 42(3), 407-417.

Yaryura-Tobias, J. A., Mancebo, M. C, \& Neziroglu, F. A. (1999). Questões teórico-clínicas do comportamento de automutilação. Revista Brasileira de Psiquiatria, 21(3), 178183. 